\title{
ANXIETY IN BONE MARROW TRANSPLANT PATIENTS
}

M. Campos, A. Olaya

Instituto Nacional de Pediatria, Mexico City, Mexico

Clinical observation has proven that anxiety disorders frequently appears in Bone Marrow transplant (BMT) patientes. It was decided to conduct a study within the psychological transplant preparation program (PTPP), where the pre-transplant anxiety level was measured. A standardized psychological test battery and a semistructured interview were conducted on a group of 50 pediatric patients ( 25 females/ 25 males, between $6-18$ years old) during the first session prior to receiving any kind of PTPP. A record was kept: Age, gender, diagnostic date, hospital stay, clinical complications and family dynamic. We corralete them with an hypothesis testing establishing a specificity level a of 0.5 and a power of 0.8 ; therefore, estimation according to the sample size, 50 patients, plus (less) $20 \%$ of losses during enrollment was done. Regarding central distribution measurements, hypothesis testing was conducted so as to compared means with paired - T.

$90 \%$ of the patients have a high anxiety level compared to their chronological age, according to what the anxiety scale evaluates. The statistical analysis showed that variables:

a) clinical complications,

b) hospital stay and

c) family dynamics were the ones that had a $90 \%$ influence in patients anxiety.

Secondly, age, gender and diagnostic date were factors that influenced the anxiety status in $64 \%$ of the cases.

It is concluded that the anxiety level in BMT patients is high from the moment they start the process, therefore, it is important to give them coping techniques and in this way prevent psychological complications in the next stages. 